\title{
Clinical significance of subcutaneous calcinosis in patients with systemic sclerosis. Does diltiazem induce its regression?
}

\author{
M Vayssairat, D Hidouche, N Abdoucheli-Baudot, J P Gaitz
}

\begin{abstract}
Objective-To establish whether diltiazem reduces subcutaneous calcinosis (SCC) in patients with systemic sclerosis (SSc), and whether this calcinosis is related to other signs or symptoms.

Methods-47 patients with SSc were evaluated and divided into two groups according to the presence or absence of SCC.

Results-Among the 12 patients with SCC who were treated with diltiazem and had sequential hand radiographs (differential time between the two radiographs: $7.8 \pm 4$ years), there was a slight radiological improvement in three patients only. More patients with SCC had anticentromere antibodies than patients without $(p=0.003)$, fewer had anti-Scl 70 antibodies $(p=0.01)$, more had telangiectasia and giant capillaries $(p=0.04$ and 0.048 respectively), and SCC patients had significantly fewer capillaries at the nailfold $(p=0.03)$. Conclusion-These results do not clearly indicate that diltiazem is effective in calcinosis associated with SSc. Among the patients with SSc, those who also had SCC exhibited a distinctive autoimmune profile and more severe cutaneous capillary injury than those without SCC.

(Ann Rheum Dis 1998;57:252-254)
\end{abstract}

Systemic sclerosis (SSc) is a multisystemic autoimmune disease of unknown aetiology. Its numerous clinical and laboratory characteristics result in a wide variety of clinical expressions, with different prognoses. Subcutaneous calcinosis (SCC) is a frequent clinical finding in SSc and its spectrum is often reported as anecdotal in SSc series. Diltiazem has been reported to reduce SCC in isolated cases. ${ }^{1-3}$ This study focused on the spectrum of calcinosis in a series of 47 patients with SSc.

\section{Methods}

The study was retrospective and included all patients with SSc evaluated in our vascular medicine unit from May 1992 to May 1996.

Unit of Vascular Medicine, Paris VI University, Hôpital Tenon, 4 rue de la Chine, 72020 Paris, France

Correspondence to: Dr Vayssairat.

Accepted for publication 11 March 1998 deposits; $2+$, multiple small areas of palpable deposits without tumoral calcifications; 3+, widespread extensive deposits with large tumoral areas, and 4+, widespread extensive deposits with large tumoral areas or skin breakdown, or both.

The study included a general and a specific scleroderma related evaluation ${ }^{6}$ with a clinical assessment of the following: area and extent of skin involvement, reflux oesophagitis, sicca syndrome, arthralgia, exertional dyspnea, and myalgia. The evaluation also included multiorgan screening that comprised chest and hand radiographs and an electrocardiogram. In addition, 46 of 47 patients had a pulmonary function test and cardiac echography, with a search for pulmonary artery hypertension. All patients were screened for antinuclear antibodies by indirect immunofluorescence, with a special search, by immunoblotting assay, for anticentromere antibodies, and antibodies to Scl70 antigen. Lastly, all patients underwent capillary microscopy, with a count of the mean number of nailfold capillaries. The results were interpreted according to a standardised method. $^{7}$

\section{STATISTICAL ANALYSIS}

Data are expressed as means (SD). The StatView Microsoft 4.02 software was used for statistical calculations. Continuous data were compared using the non-parametric MannWhitney U test, and discrete data, using Fisher's exact test.

\section{Results}

CLINICAL ASPECTS OF SCC

Forty seven patients with SSc were included. They comprised 41 women and six men whose mean age was 56 (13) years. There were 29 patients with limited SSc, and 18 with diffuse SSc. Table 1 shows the demographic data and the results of the clinical and paraclinical evaluations.

Twenty three patients (49\%) had SCC. It was located in the digits in 21 cases, and elsewhere in six cases (four patients had it in both locations). For 16 of these 23 patients, the classification of Berger could be recorded reliably: five of the 16 were classified as $1+$, four as $2+$, one as $3+$, and six as 4+. Fifteen of the 29 patients with limited SSc $(51 \%)$ and eight of the $18(44 \%)$ with diffuse SSc had SCC, that is, 23 in all. Furthermore, in patients with SCC, the mean duration of SSc was 11.9 (4) years, and did not differ from the mean duration in patients without SCC, which was 11.5 (7) years, $\mathrm{p}=0.7$. 
Table 1 Demographic data and results of the clinical and paraclinical evaluations in a series of 47 patients with systemic sclerosis

\begin{tabular}{ll}
\hline Parameter & \\
\hline Age at diagnosis of SSc (y) & $44(12)(24-67)$ \\
Age at the most recent follow up & $56(13)(29-83)$ \\
Sex ratio: & $\mathrm{F}=41, \mathrm{M}=6$ \\
Classification & Limited=29, Diffuse $=18$ \\
Limited/diffuse & $7.7(2)(4-13)$ \\
number of capillaries per mm at the nailfold & \\
Presence of & $47 / 47(100)$ \\
Raynaud's phenomenon & $46 / 46(100)$ \\
Abnormal capillaroscopy & $45 / 47(95)$ \\
Antinuclear antibodies & $38 / 46(82)$ \\
Giant capillaries & $36 / 46(78)$ \\
Telangiectasia & $36 / 47(76)$ \\
Oesophagitis & $36 / 47(76)$ \\
Sicca syndrome & $35 / 47(74)$ \\
Facial skin involvement & $35 / 47(74)$ \\
Digital necrosis & $34 / 47(72)$ \\
Polyarthralgia & $27 / 46(58)$ \\
Abnormal CO transfer & $23 / 44(52)$ \\
Pitting scars & $22 / 43(51)$ \\
Osteolysis & $23 / 47(49)$ \\
Calcinosis & $20 / 47(42)$ \\
Anticentromere antibodies & $17 / 45(37)$ \\
Pulmonary fibrosis & $15 / 47(32)$ \\
Anti-Scl 70 antibodies & $14 / 46(30)$ \\
Pulmonary hypertension & $10 / 47(21)$ \\
Myositis & $10 / 46(21)$ \\
Restrictive lung syndrome & $7 / 47(14)$ \\
Truncal skin involvement & $7 / 47(15)$ \\
Heart involvement & $4 / 47(8.5)$ \\
Digital amputation & $2 / 47(4)$ \\
Renal involvement & \\
& \\
\hline & \\
Except when other & \\
&
\end{tabular}

Except when otherwise specified, continuous data are expressed as mean (SD) (ranges), and discrete data, as the number of patients with the positive item out of the number of patients tested (\%)

The immunological profile differed in patients with SCC and those without. Thus, 15 of the 23 patients with SCC (65\%) had anticentromere antibodies compared with five of the 24 patients without $(20.8 \%, \mathrm{p}=0.003)$, and three of the 23 patients with SCC (13\%) had anti-Scl 70 antibodies compared with 12 of the 24 patients without $(50 \%, \mathrm{p}=0.01)$.

Capillary injury involvement was greater in patients with SCC than in those without. Thus, more patients with SCC than without had telangiectasia $(91 \% v 65, \mathrm{p}=0.04)$, and digital tip necrosis $(87 \%$ v 62.5, $\mathrm{p}=0.09)$, but the latter difference was not significant. In addition, more patients with SCC had giant capillaries, (95\% $v 70.8, \mathrm{p}=0.048)$ and a reduced number of capillaries per millimeter at the nailfold (7 (1.7) $v 8.4(2) \mathrm{p}=0.03)$.

\section{CHANGES OBSERVED DURING DILTIAZEM} TREATMENT

All 23 patients with SCC were prescribed diltiazem for the treatment of their associated Raynaud's phenomenon at a dose of $60 \mathrm{mg}$

Table 2 Evolution of the hand radiological findings in a series of 12 systemic sclerosis patients treated with diltiazem

\begin{tabular}{llll}
\hline Patient & $\begin{array}{l}\text { Duration of diltiazem } \\
\text { treatment }(y)\end{array}$ & $\begin{array}{l}\text { Duration of radiographic } \\
\text { follow up }(y)\end{array}$ & Change in calcinosis \\
\hline 1 & 6 & 3 & slight decrease \\
2 & 5 & 3 & no change \\
3 & 1 & 1 & no change \\
4 & 7 & 6 & increase \\
5 & 7 & 8 & slight decrease \\
6 & 11 & 4 & no change \\
7 & unknown & 9 & no change \\
8 & 13 & 11 & increase \\
9 & 4 & 2 & no change \\
10 & 1 & 4 & no change \\
11 & 15 & 12 & increase \\
12 & 10 & 15 & slight decrease \\
\hline
\end{tabular}

three times a day. All patients tolerated the mild side effects of diltiazem. Three patients with SCC improved after surgical exeresis, and one patient's SCC resolved spontaneously, after a local infection with ulceration. In 12 patients, iterative hand radiographs allowed precise comparisons throughout a mean follow up of 6.5 (4) years (range: 1-15), accounting for 80 patient years of treatment (table 2). SCC remained stable in this series of 12 patients; it was strictly unchanged in six of them, regressed slightly in three, and intensified slightly in three. For the three patients who improved, the summed period of treatment with diltiazem was 23 years, and for those whose SCC intensified, 35 years.

\section{Discussion}

The scientific evidence in favour of a positive effect of diltiazem on SCC in patients with SSc is based on three publications, but together they only account for five patients. ${ }^{1-3}$ In the present retrospective uncontrolled study of 23 patients with a summed long duration of diltiazem treatment, we do not confirm that this treatment indeed had a positive effect. Nevertheless, our results do not exclude the possibility that diltiazem may be effective in a small group of patients. However, the negative results for this series have to be examined as a function of the daily dose of diltiazem, which was $180 \mathrm{mg}$ in our study, compared with 240 to $480 \mathrm{mg}$ for authors who reported a positive effect. ${ }^{1-3}$ Furthermore, all the patients in this series had a long term prescription for diltiazem because it had a beneficial effect on their Raynaud's phenomenon, and therefore our results did not support the hypothesis that there is a relation between improved digital perfusion and calcinosis.

Calcinosis is a frequent finding in patients with SSc and its presence is not restricted to patients with the limited form of the disease. We found that patients with SCC exhibited a distinct immunological profile, that more of them had anticentromere antibodies than those without SCC, and that fewer of them had antiScl 70 antibodies. These findings are in agreement with what is known about limited Ssc, ${ }^{8-10}$ in which anticentromere antibodies are more frequent than anti-Scl antibodies.

As far as we know, this study is the first in which capillary cutaneous injury was found to be more severe in the presence of SCC than in its absence. Not only did more SCC patients have telangiectasia, giant capillaries, and fewer capillaries at the nailfold, but the history of digital necrosis was also more frequent in these patients, a finding already reported for patients with anticentromere antibodies. ${ }^{10}$

Taken together, our findings show that patients with SSc associated with SCC exhibit a distinct immunological profile and more marked cutaneous capillary injury involvement than patients without subcutaneous calcinosis. For all these reasons, we recommend hand radiographs for routine evaluation of SCC in patients with SSc, as it is an easily detected, inexpensively obtained clinical finding related to the severity of SSc microangiopathy. 
1 Farah MJ, Palmieri GMA, Sebes JI, Cremer MA, Massie JD, Pinals RS. The effect of diltiazem on calcinosis in a patient with the CREST

2 Dolan AL, Kassimos D, Gibson T, Kingsley GH. Diltiazem induces remission of calcinosis in scleroderma. $\mathrm{Br} \mathrm{J}$ Rheumatol 1995;34:576-8.

3 Palmieri GMA, Sebes JI, Aelion JA, Moinuddin M, Ray $\mathrm{MW}$, Wood GC, et al. Treatment of calcinosis with diltiazem. Arthritis Rheum 1995;38:1646-54.

4 LeRoy EC, Black C, Fleischmajer R, Jablonska S, Krieg T, Medsger TA, et al. Scleroderma (systemic sclerosis): classification, subsets and pathogenesis. J Rheumatol 1988;15: 202-5.

5 Berger RG, Featherstone GL, Raasch RH, McCartney WH, Hadler NM. Treatment of calcinosis universalis with low-dose warfarin. Am J Med 1987;8372-6.

6 Vayssairat M, Baudot N, Abuaf N, Johanet C. Long-term follow-up study of 164 patients with definite systemic sclerosis: classification considerations. Clin Rheum 1992; $11: 356-63$

7 Garnier F, Vayssairat M, Priollet P, Housset E. Nailfold capillary microscopy in mixed connective tissue disease: comparison with systemic sclerosis and systemic lupus erythematosus. Arthritis Rheum 1986;29:189-95.

8 Fritzler MJ, Kinsella MJ, Garbutt E. The CREST syndrome: a distinct serologic entity with anticentromere syndrome: a distinct serologic entity with
antibodies. Am J Med 1980;69:520-6.

9 Steen VD, Powell DL, Medsger TA. Clinical correlations and prognosis based on serum autoantibodies in patients with systemic sclerosis. Arthritis Rheum 1988;31:196-203.

10 Wigley FM, Wise RA, Miller R, Needleman BW, Spence RJ. Anticentromere antibody as a predictor of digital ischemic loss in patients with systemic sclerosis. Arthritis Rheum 1992;35:688-93. 